\title{
A "One Health" surveillance and control of brucellosis in developing countries: Moving away from improvisation
}

\author{
Jacques Godfroid $^{\text {ab* }}$, Sascha Al Dahouk ${ }^{\text {cd }}$, Georgios Pappas ${ }^{\mathrm{e}}$, Felix Roth ${ }^{\mathrm{f}}$, Gift Matope ${ }^{\mathrm{g}}$, John \\ Muma $^{\mathrm{h}}$, Tanguy Marcotty ${ }^{\text {ib }}$, Dirk Pfeifferj, Eystein Skjerve ${ }^{\mathrm{k}}$ \\ ${ }^{a}$ Department of Food Safety and Infection Biology, Norwegian School of Veterinary Science, Tromsø, Norway \\ ${ }^{\mathrm{b}}$ Department of Veterinary Tropical Diseases, Faculty of Veterinary Science, University of Pretoria, Onderstepoort, South Africa \\ ${ }^{\mathrm{c}}$ Federal Institute for Risk Assessment, Berlin, Germany \\ ${ }^{\mathrm{d}}$ RWTH Aachen University, Department of Internal Medicine III, Aachen, Germany \\ ${ }^{\mathrm{e}}$ Institute of Continuing Medical Education of Ioannina, Ioannina, Greece \\ ${ }^{\mathrm{f}}$ Swiss Tropical Institute, Basle, Switzerland \\ ${ }^{\mathrm{g}}$ Department of Paraclinical Veterinary Studies, University of Zimbabwe, Harare, Zimbabwe \\ ${ }^{\mathrm{h}}$ Department of Disease Control, University of Zambia, School of Veterinary Medicine, Lusaka, Zambia \\ ${ }^{\mathrm{i}}$ Institute of Tropical Medicine, Antwerp, Belgium \\ j Veterinary Epidemiology \& Public Health Group, Department of Veterinary Clinical Sciences, Royal Veterinary College, London, UK \\ ${ }^{k}$ Department of Food Safety and Infection Biology, Epicenter, Norwegian School of Veterinary Science, Oslo, Norway
}

\begin{abstract}
* Corresponding author at: Norwegian School of Veterinary Science, Department of Food Safety and Infection Biology, Section of Arctic Biology, Stakkevollveien 23,9010Tromsø, Norway. Tel.: +47 776654 21; fax: +47 77694911.

E-mail address: Jacques.godfroid@nvh.no (J. Godfroid).
\end{abstract}

\begin{abstract}
Although a "One Health" approach has been successfully implemented for emerging infectious zoonotic diseases with pandemic potential, we still lack a conceptual framework to address enzootic diseases like brucellosis. The vast majority of published brucellosis studies in the developing world rely solely on serology. An important shortcoming of brucellosis serology is the impossibility to infer which (smooth) Brucella spp. induced antibodies in the host. In this respect, mixed farming and especially raising small ruminants along with cattle, a common practice in the developing world, is reported to be a risk factor and a central question that has to be answered is whether cattle are infected with $B$. melitensis or with B. abortus or with both Brucella species. Therefore the isolation, identification and molecular characterization of Brucella spp. in human and the different livestock species needs to be undertaken to define a sound conceptual framework, identify the source of infection and plan appropriate control measures.
\end{abstract}

Keywords:

Brucellosis ; One Health ; Zoonoses ; Epidemiology ; Serology ; Mixed herds ; Nomadism ; Vaccination ; Control ; Eradication

\section{Introduction}

In its foreword of the book "People, Pathogens, and Our Planet" - Volume 1: Towards a One Health Approach for Controlling Zoonotic Diseases, Juergen Voegele, Director of Agriculture and Rural Development, The World Bank writes the following: "A global surveillance and control system that is established primarily for emerging infectious zoonotic diseases with pandemic potential can be readily improvised to address the endemic diseases that are a priority in many developing countries, few of which have the capacity or resources necessary to monitor or control them effectively" (http://siteresources.worldbank.org/INTARD/Resources/PPPWeb.pdf).

In other words, to date there is no "One Health" surveillance and control system for endemic (i.e., enzootic) diseases like brucellosis, particularly - but not exclusively - in developing countries. Such a strong statement contradicts our intuitive feeling that there are benefits for public health and society to be gained by implementing sound control and eradication brucellosis programs for livestock, although such benefits need to be demonstrated, particularly in countries with scarce resources [1]. Should such benefits be documented, does this however mean that they are the outcome of a conceptually sound "One Health" approach?

It is fair to say that there are many unknowns and misconceptions that may lead to the implementation of improvised control measures for endemic diseases as written by Juergen Voegele. It is important to stress that there is an inherent risk that improvised measures might beat best not justified and would not help in providing a sustainable solution or worse, improvisation may be counter-productive or even detrimental. In order to be successful, a "One Health" approach has to be truly multidisciplinary and every component of a global/holistic approach has to be addressed proficiently in its own right. More, given the changes in the livestock sector, its contact with wildlife and the resurgence and emergence of zoonotic diseases linked to it, a new "One Health" research and policy-generation strategy has to be defined [2]. It is in this context that the World Animal Health Organization (OIE) is endorsing a "One Health" approach which will result in a deeper and sustainable political support for the coordinated prevention of high public health and animal impact diseases at the human-animal interface (http://www.oie.int/en/for-the-media/onehealth/).

The aim of the manuscript is to highlight some of the unanswered questions related to the biology of Brucella spp. infections in humans and livestock, as well as questions related to brucellosis control measures that besides being efficient should also be realistic in developing countries where human and financial resources are scarce. Such information is needed for the definition of a "One Health" conceptual framework for zoonotic brucellosis in the developing world.

Control and eradication programs of animal brucellosis are implemented in the developed world whereas resources are often 
not allocated to such programs is developing countries. Epidemiological studies in these countries rely almost exclusively on brucellosis serology. Shortcomings of serology will be highlighted by reviewing scientific publications from Uganda, one of the few developing countries were brucellosis has been extensively studied for the last 15 years. From the human health perspective, the benefit of mass vaccination of livestock and the dramatic public health consequences following the absence or the discontinuation of animal vaccination campaigns will be illustrated by situations prevailing in Mongolia and Greece. Risk factors related to husbandry practices like nomadism and mixed herds will be addressed and the importance of non-classical livestock species and wildlife as a source of Brucella spp. for people will be highlighted. Finally, recommendations related to targeted actions will be made in the context of a sound conceptual brucellosis "One Health" approach.

\section{Brucellosis control and eradication programs}

Currently, about half a million human brucellosis cases are annually reported worldwide but the estimated number of unreported cases due to the unspecific clinical symptoms of the disease is supposed to be 10 times higher. In endemic countries prevalence rates often exceed 10 cases per 100,000 population [3]. Brucellosis is transmitted to humans from direct contact with livestock (occupational disease for abattoir personnel, farmers and veterinarians for example) or more often by ingestion of unpasteurized milk or milk products [4]. In heifers that aborted, B. abortus is found in the uterus, in milk, in the mammary glands and associated lymph nodes [5]. Of significant epidemiological importance, B. abortus was also found in weak and healthy calves born from experimentally infected heifers [5].

The consumption of cattle, sheep and goat meat does not seem to play a role, although meat from animals that appear to be sick at the time of slaughter should not be consumed [6]. However, bacteria can be transmitted to humans by unsafe butchering and consumption of under-cooked meat. A recent report from Botswana suggests that household bush meat processing practices represent a significant Brucella spp. exposure risk to family members and the community [7].

Person to person transmission of brucellosis through breast feeding or by sexual intercourse, although reported [8] is epidemiologically anecdotal and therefore brucellosis in humans almost always originates from an animal reservoir and results from different risk factors and behavioral traits $[1,4]$.

In the developed world, for more than four decades, control and eradication programs of brucellosis in livestock have been implemented by national veterinary services. Classically after a first phase in which the infection is controlled by compulsory vaccination, then vaccination is gradually restricted and eventually prohibited whereas a "test and slaughter" policy is implemented in order to eradicate the infection. More than a decade is usually needed to complete the brucellosis eradication program by a "test-and-slaughter" policy and key for success is a sufficient financial compensation scheme for farmers for their culled livestock. In the European Union (EU), such national programs are co-financed by the EU and the Member States (MSs). This policy has been successfully implemented for bovine as well as ovine and caprine brucellosis in Northern MSs (with the notable exception of bovine brucellosis in the United Kingdom), whereas eradication programs, particularly ovine and caprine brucellosis eradication programs are not yet completed in some Southern European MSs (http://ec.europa.eu/food/animal/diseases/eradication/eradication_bovine_sheep_goats_brucellosis_en.pdf).

Countries are reporting on the national animal health situation to the OIE via the World Animal Health Information Database (WAHID) Interface. This interface provides access to all data held within OIE and can be accessed following the link: http://web.oie.int/wahis/public.php?page=home. Unfortunately, the information related to brucellosis that is provided by some developing countries is scarce or absent. In such resource poor countries, the implementation of an efficient eradication policy is impossible and thus innovative approaches taking into account the scarcity of financial resources as well as the perceptions and attitudes of communities have to be defined where human brucellosis is documented to be a public health problem [9]. One example of such an innovative approach has been studied in Mongolia where the economic benefit, cost-effectiveness, and distribution of benefit of improving human health through the control of brucellosis by mass vaccination of livestock has been estimated [10,11]. In Tajikistan biannual conjunctival vaccination of small ruminants with Rev 1 reduced the seroprevalence by 80 per cent in 5 years and the prevalence of households with evidence of infection in their animals dropped from 25.1 to 7.5 per cent [12]. However, the extent to which this success is to be attributed to the implementation of a conceptually sound "One Health" framework remains to be analyzed. Interestingly, in Nigeria, the most populated country in Africa, from a medical perspective, brucellosis has been classified as a sporadic zoonosis and it is therefore unlikely that specific veterinary public health measures will be prioritized, even more so given that links between medical and veterinary officers in Nigeria can be said to be non-existent or at best very weak [13]. Lastly, in the scientific literature related to human brucellosis in Sub-Saharan Africa, prevalence rates are based on serological results, with less than a handful reports over the last four decades on the isolation of any Brucella strain in patients. The absence of isolation of any Brucella spp. inducing seropositivity and disease in patients makes it difficult to trace back the origin of the infection when brucellosis seropositivity is detected in different species of the animal reservoir [14].

\section{Shortcomings in brucellosis serology}

The "Manual of Diagnostic Tests and Vaccines for Terrestrial Animals" published by the OIE lists diagnostic tests in two categories: "prescribed" and "alternative". Prescribed tests are required by the OIE Terrestrial Animal Health Code for the international movement of animals and animal products and are considered optimal for determining the health status of animals. The manual can be consulted on line following the link: http:/www.oie.int/en/international-standard-setting/ terrestrialmanual/access-online/. 
As recommended by the OIE, such tests have to be validated and antigens should be purchased from reliable sources. A control certificate of the purchased batch issued by a competent control authority should always be provided. Using tests that do not meet these requirements may generate unreliable results and may thus lead to wrong epidemiological inferences.

The OIE puts the emphasis on the use of prescribed tests for trade in order to avoid transmission across borders. Those tests are thus aiming at providing additional guaranties on the absence of Brucella infection in traded animals. Trading occurs nowadays mainly between countries that are "officially-free" or alternatively, at least the herd/flock of origin must be recognized to be brucellosis-free. However, traded animals can be recently infected but not yet detected or can get infected during transport or when mingling at market places. This means that tests recommended for trade are those able to detect recent (acute) infection in animals. The Rose Bengal Test (RBT) is usually used as a detection test, and if a serum sample is classified RBT positive, the result is usually confirmed by performing a Complement Fixation Test (CFT, which is a test difficult to standardize and perform) or preferably an ELISA (commercial kits controlled by OIE reference laboratories are available) on the same serum sample (tests are said to be applied in series). It has to be noted that bacteriology is the only diagnosis of certainty.

It has been shown under experimental conditions that RBT negative heifers which are chronically infected with $B$. abortus are detected by ELISAs and thus in order to detect the majority of the chronically infected animals, it has been suggested to using both tests in parallel or alternatively only to using ELISAs $[15,16]$. However, costs linked to purchasing antigens make the RBT the preferred detection method worldwide for early detection as in enzootic situations.

It is worth to be reminded that vaccination with the vaccines S19 and Rev 1, in cattle and small ruminants respectively, induces antibodies that are detected in brucellosis serological tests. This complicates the interpretation of serological results when vaccination is implemented. Therefore the use of the brucellosis vaccine RB51 has been advocated in cattle given that this vaccine will not induce post-vaccination antibodies in the OIE prescribed brucellosis serological tests [17].

Another important and often forgotten shortcoming of brucellosis serology is the impossibility to infer which (smooth) Brucella spp. induced antibodies in the animal or human host [14]. Indeed, the Brucella Smooth Lipopolyssacharide (S-LPS) carries the immunodominant epitopes that are shared by $B$. abortus, $B$. melitensis and $B$. suis and virtually all serological tests for detecting anti S-LPS antibodies (like the OIE prescribed tests) use $B$. abortus antigens [18]. The preferentail hosts of $B$. abortus, B. melitensis and B. suis and the pathogenicity for humans of their respective biovars are depicted in Table 1.

Table 1

Brucellaabortus, B. melitensis and B. suis, biovars, preferential hosts and pathogenicity for humans.

\begin{tabular}{llll}
\hline Brucella species & Biovars & Preferential host(s) & Pathogenicity for humans \\
\hline B. abortus & $1-6,9$ & Cattle & High \\
B. melitensis & $1-3$ & Sheep, goat & High \\
B. suis & 1,3 & Pig & High \\
& 2 & Wild boar, hare & Low \\
& 4 & Reindeer, caribou & High \\
\hline
\end{tabular}

a Only three cases have been reported in France: one in a farmer having a small herd of outdoor reared pigs and two cases in wildboar hunters.

As a consequence, infection in humans with B. abortus, B. melitensis or B. suis will induce the same serological pattern and antibodies induced by these different Brucella species are actually detected by the same serological tests. The obvious consequence is that sound epidemiological inferences are very difficult to make in the presence of seropositivity in animals of multiple species. Such shortcomings will be highlighted hereafter from serological brucellosis studies performed in Uganda, a country where brucellosis has been widely studied for the last 15 years.

\subsection{Brucellosis studies in Uganda (1998-2011)}

At the turn of the 21st century, prevalence and risk factors for Brucella seropositivity in goats in eastern and western Uganda were investigated [19]. The authors suggested that it was predominately, but not exclusively, B. melitensis that induced antibodies in goats, although no attempt to isolate Brucella strains was done. The authors made these inferences based on the combined use of a Card Test (CT, a buffered brucellosis agglutination test equivalent to the RBT), and a Tube Agglutination Test (TAT). While the CT is a prescribed test and uses B. abortus antigen, the TAT uses B. melitensis as antigen and is not an OIE prescribed or alternative test. This TAT does not meet the minimal requirements as laid down in the OIE manual and is thus not recommended by the OIE. Notwithstanding these shortcomings, goats should be considered as a reservoir of Brucella spp. for humans and other animal species. Several other publications from Uganda point towards dairy cattle as being infected with $B$. abortus, although no strain isolation is reported [20-23]. Based on serological results, poor correlation between the distribution of human brucellosis cases in urban and peri-urban areas of Kampala and the distribution of cattle was found, suggesting that most of the human brucellosis cases resulted from consumption of raw milk transported from peri-urban and rural areas of Kampala and/or dairy production areas outside Kampala [24,25]. The B. abortus biovar 3 reference strain (Tulya) was originally isolated from a man in 1958 by Dr. K.W.Jones, in Kampala, Uganda [26]. The sole documented B. abortus isolation from cattle in Uganda has been reported in the literature in 2012 [27].

Many unanswered questions remain to be studied in Uganda. First, attempts to isolate Brucella strains should be performed in different animal species, given that seropositivity has been documented in cattle, pig, goat and (unspecified) wildlife in a 10-year (1998-2008) retrospective study [28]. The fact that B. melitensis has been isolated from cattle besides B. abortus in neighboring 
Kenya [27] highlights the importance of isolating and identifying Brucella strains in order to trace back the reservoir species, not the spill over species. Indeed, all the reported cases of B. melitensis infection in cattle are linked to a reservoir of $B$. melitensis in small ruminants [29,30] and bovines are not considered to be maintenance host for B. melitensis [14]. Second, in a serological study performed in cattle, $2 / 9$ herds (in which seropositive animals were found) were vaccinated and thus seropositivity may have been induced by vaccination more than by infection with wildtype Brucella spp. [21]. The cattle herd prevalence may thus be overestimated by more than $20 \%$ in this study. Therefore, information on the vaccination status of animals is of paramount importance in order to make a sound interpretation of serological results. Third, it is wrong to state that milk has been found infected with Brucella based on positive Milk Ring Test (MRT) results [24]. Indeed, only the presence of anti-Bruce/fa antibodies is detected in milk by the MRT and such antibodies may be induced by vaccination and/or infection with wildtype Brucella spp. The actual question remaining to be addressed is to which extend vaccination will prevent the excretion of wildtype Brucella spp. in milk, regardless of the MRT result, notwithstanding the fact that vaccine strains may be excreted in milk too. Four, all Brucella spp. susceptible livestock species should be taken into account to get insight into the global brucellosis epidemiological picture. In this respect, pig is an important livestock species, at least in some parts of Uganda and its importance as a reservoir of Brucella spp. including B. suis needs to be addressed, since high sero-prevalences have been found in pigs [28], notwithstanding that to date, according to the OIE, none of the serological tests has been shown to be reliable in routine brucellosis diagnosis in individual pigs, because pigs are often infected with Yersinia enterocolitica 0:9 [31]. It is striking that no isolation of B. suis has been reported in Sub-Saharan Africa although brucellosis in pigs is thought to be prevalent [32]. Five, no isolation of Brucella spp. from human patients in Uganda has been documented in the international literature since 1958. In humans, Brucella S-LPS cross-reactivity with Francisella tularensis, Yersinia enterocolitica 0:9 and Vibrio cholerae, is a potential source of unspecific results in brucellosis serological tests, such as the RBT, CFT and ELISA [33]. However the extent to which cross reactive bacteria induce false positive serological reactions in humans in Sub-Saharan Africa stills needs to be addressed.

Given the overall shortcomings of brucellosis serological tests, the isolation, identification and molecular characterization of Brucella spp. in human and the different livestock species needs to be undertaken; otherwise describing human brucellosis as under-reported and highlighting the benefits of control measures such as vaccination in different animal species may be speculative and misleading.

\section{Mass vaccination against animal brucellosis and human health benefits}

The benefits of the implementation of mass vaccination campaigns as well as the negative consequences of the unsuccessful implementation or the discontinuation of such campaigns will be illustrated by the Mongolian and the Greek examples.

\subsection{Cattle and small ruminants mass vaccination in Mongolia (2000-2002)}

A dynamic model of livestock-to-human brucellosis transmission in Mongolia has been defined and transmission within sheep and cattle populations and transmission to humans are part of the model [11]. The authors acknowledge that some parameters were not integrated in the model because of the lack of data. For example, the transmission between goats and from goats to humans was omitted. Likewise brucellosis in yaks (Bos grunniens) and camels was not accounted for. The simultaneously fitted sheep-human and cattle-human contact rates show that $90 \%$ of human brucellosis was small-ruminant derived. The authors report that only B. melitensis has been isolated from human patients. Based on this information, those human cases related to cattle contacts have resulted in $B$. melitensis infection. It is worth reiterating that contact between small ruminants and cattle is almost always described in the literature as the source of $B$. melitensis infections in cattle $[29,30]$ and thus bovines are not considered to be maintenance host for B. melitensis [14]. Unfortunately, transmission between small ruminants and cattle was omitted in the afore-mentioned model [11]. Given the central role of sheep and goats in the contamination of bovines and humans, allocating additional resources for the control of brucellosis in sheep by discontinuing vaccination campaigns in cattle, for example, could be considered in the future. Notwithstanding the imperfections of the model, the cross-sector societal economic assessment of the profitability of brucellosis mass vaccination in Mongolia showed that the societal benefits were three times higher than the cost of the intervention [10]. Unfortunately, recent data suggests that livestock mass vaccination campaigns were not successfully implemented in two representative Provinces (Sukhbataar and Zavkhan "Aimags"). Indeed, a cross-sectional study performed in June and August 2010 showed that brucellosis seroprevalences in herders and herder families were high, suggesting that human brucellosis remains a huge public health problem in Mongolia (oral communication by Selenge Tsen, Brucellosis 2011 International Research Conference, September 21-23, Buenos Aires, Argentina).

The question whether $B$. melitensis is the only zoonotic Brucella species present in livestock in Mongolia, although only $B$. melitensis infections are reported in human patients still awaits an answer. Indeed, in the neighboring Inner Mongolia province of China, human brucellosis cases have been described. The vast majority of isolates were B. melitensis although $B$. abortus has been isolated in some districts too [34]. It is interesting to note that, from a public health point of view, there is no mention of a "One Health" approach in this publication and the authors suggest allocating resources to high-risk districts to strengthen active surveillance in humans, not in animals.

\subsection{Ovine and caprine eradication program in Greece at the turn of the 21st century}

In Greece, small ruminant farming is the largest sector of food-animal production with more than 11 million animals and the goat population is the largest in the EU [35]. Ovine and caprine brucellosis control program started in 1975, based on vaccination of young animals (3-6 months old) to be kept as replacements. It is believed that the most important factor that contributed to the 
significant decrease of incidence of human brucellosis was milk pasteurization that was started in Greece at the same time as well as improved sanitary measures taken during the production of feta cheese. This vaccination strategy was implemented until the end of 1994, when based on its success the Greek veterinary authorities thought that the eradication of ovine and caprine brucellosis was feasible. The brucellosis vaccination campaign of young animals was thus stopped and a test-and-slaughter policy was started in 1993. At the end of 1998, the test-and-slaughter policy was abandoned and an emergency mass vaccination campaign of both young and adult animals against brucellosis was started because of (1) a re-emergence of brucellosis in small ruminants and (2) an increase of occupational brucellosis (pasteurization of milk had previously dramatically reduced the foodborne origin of brucellosis in Greece) [35].

Eradication of ovine and caprine brucellosis seems thus to be very difficult to achieve and is likely beyond reach in the developing world. Unsuccessful programs may have dramatic consequences for both public health and the food producing sector. Therefore, the implementation of sanitary measures such as the pasteurization of milk and cheese as well as mass vaccination are to be advocated in resource poor countries in order to contribute significantly to lower incidence of human cases.

\section{Mixed herds an nomadism}

Intermingling of livestock species may cause uninfected animals to easily get exposed to the disease from multiple sources such as abortion discharges and direct contact with infected animals. Mixed farming and especially raising sheep and/or goats along with cattle was reported by many researchers to be a risk factor for Brucella transmission between different animal species. However this transmission does not occur indifferently in both directions. Indeed, whereas infection of sheep and goats with $B$. abortus is seldom reported [36], B. melitensis infection in cattle has been reported where B. melitensis was found in its preferential hosts, i.e., sheep and goats in France [29] and in Spain [30]. The importance of this in the developed world is illustrated by a recent study in Egypt where cattle and buffalo kept in a household with sheep and goats had 6.32 times the odds of testing seropositive for Brucella spp., compared to cattle and water buffalo (Bubalus bubalis) that were not [37]. Given this information and given that B. melitensis is currently the most common Brucella species isolated in human patients in Egypt, it is urgent to assess to which extend B. abortus is a veterinary public health problem in Egypt and by extension in the region as well as in whole Africa.

There is no data on the prevalence of ovine and caprine brucellosis within a flock in the absence of centrally organized control measures. Seroprevalence rates vary greatly and epidemiological information is often missing for sound inferences [37]. This is even more the case for livestock herded under nomadic conditions. It is worth to stress that nomadism does not mean absence of control measures. For example, differences in brucellosis prevalence have been reported in pastoral areas of Ethiopia. Such differences might be due to the variation in herding practices. In the Afar region, mixing animals from the various areas is common at communal grazing and watering areas. In the Somali region, only animals belonging to a given clan are allowed to be mixed, and there is a strong clan-based segregation of animals and use of rangeland [38].

The epidemiology of brucellosis in free roaming pigs is different. Indeed, it has recently been demonstrated that feral pigs can act as reservoir hosts of $B$. abortus in the absence of contact with cattle for more than 25 years [39]. These findings stress the importance of identifying which Brucella species is infecting pigs in Sub-Saharan Africa, where no reliable information on porcine brucellosis is currently available.

\section{Brucellosis in non-classical livestock species, in wildlife and at the wildlife/livestock/human interface}

Besides B. suis biovar 4 infection in reindeer/caribou (Tarandus tarandus) and Brucella suis biovar 2 infection in wildboar (Sus scrofa), brucellosis in wild ungulates seems always to result from a spillover from a livestock reservoir [40]. Only in very few cases does the infection establish itself and become sustainable in wildlife spillover species. This results almost inevitably from wildlife management practices as suggested for winter feeding of elk (Cervus elaphus) and bison (Bison bison) in the Yellow Stone Conservation Area [41]. Another example is Brucella suis biovar 1 infection in feral pigs, in the South Eastern States of the United States. The escape or release of infected pigs resulted in a wide spread of the infections and occupational/recreation infection in hunters [39].

Chronic clinical brucellosis, i.e., hygroma at the carpal joint, has been observed in different wildlife species in Africa, particularly in buffalo (Syncerus caffer) [42]. Buffalo, is utilized both legally and illegally as a source of bush meat. It has recently been suggested in Botswana, that household bush meat processing practices can provide Brucella spp. exposure risk to family members and the community and that public health officials need to be alert to the possibility of human infections arising from the use of bush meat [7]. From a medical point of view B. suis biovar 4 infection in reindeer, caribou and moose (Alces alces) in the Canadian and Russian Arctic, as well as in Alaska is important. These animals are part of the subsistence lifestyle and harvesting of native communities in the Arctic for whom B. suis biovar 4 infections have been documented [43,44].

Camelids represent by far the most non classical livestock species. The home ranges of dromedary (Camelus dromedaries) are the hot and dry regions of North Africa, Ethiopia, the Near East and West-Central Asia, whereas the bactrian camel (Camelus bactrianus) occupies the cold deserts of southern areas of the former Soviet Union, Mongolia, East-Central Asia and China. The habitat of the limeades (genus Lama) are the cold heights of Latin America [45]. Camelids can be infected with both B. melitensis and B. abortus $[45,46]$. 


\section{Discussion}

Nearly two-thirds of human pathogens are zoonotic and, of greater concern, nearly three-quarters of emerging and re-emerging diseases of human beings are zoonoses [47]. This is the rationale for establishing a global surveillance and control system primarily for emerging infectious zoonotic diseases with pandemic potential. However, improvising a system based on the early detection of infectious zoonotic diseases with pandemic potential to assess the zoonotic risk of endemic diseases prevailing in developing countries seems not to be appropriate as highlighted by the analysis of different brucellosis epidemiological situations.

Both approaches are in essence different and need to be addressed according to different conceptual frameworks. In this respect it was striking that during the first "One Health" conference held in Melbourne in February 2011, during the panel discussion on the definition of a global "One Health" concept, no consensus could be reached as to what such a global concept would encompass. It is important to acknowledge that "virus hunting", "foodborne zoonoses" and "neglected zoonoses" are different issues, each of these needing a sound and specific conceptual framework. If such a framework has been defined and is nowadays successfully implemented for emerging infectious zoonotic diseases with pandemic potential, this is not the case for endemic and often neglected zoonoses, like brucellosis.

For livestock diseases, the OIE rightly puts the emphasis on avoiding transmitting diseases while trading live animals. Hence it is important to implement a standardized early detection system worldwide. The OIE prescribed also alternative tests which are methods considered being suitable for the diagnosis of disease in a local situation, such tests may be the most appropriate for use in the developing world in the context of subsistence farming. Indeed, in developing countries where brucellosis is enzootic, the emphasis should not be put on the early detection of a new Brucella spp. infection but rather on identifying those animal species acting as reservoir of Brucella spp. and being an important source of brucellosis for humans.

Studies performed in Sub-Saharan Africa suggest that cattle are a significant source of Brucella spp. for humans, if not the most important one. It remains to be known if cattle are mainly infected with B. melitensis (which is documented in North Africa [48]) or with B. abortus like documented in Zimbabwe [49], or with both Brucella species like recently described in Kenya [27]. The fact that $B$. abortus infected cattle may be the main source of brucellosis for people in Sub-Saharan Africa means that comparison with the situation prevailing in the Middle East, where small ruminants are the main reservoir of B. melitensis, is not valid. We therefore need a new conceptual framework to address this issue in an operational "One Health" brucellosis perspective for Africa, where people may get infected by small ruminants and cattle infected with B. melitensis like in Egypt [48] as well as for countries like Zimbabwe, where people may get infected predominantly by $B$. abortus infected cattle. In this later case, control methods in cattle maybe more easily implemented as in small ruminants notwithstanding that the pathogenicity of B. abortus in humans is not known to the extent of B. melitensis [50], although the treatment of human patients advocated by the WHO is the same regardless of the Brucella species [51].

It is also worth mentioning that B. abortus and B. melitensis have been detected in supramammary lymph nodes in camels in Darfur, Western Sudan and this presents a potential hazard to those who consume raw camels' milk, a common practice in nomadic camel owners [46].

Lastly, the true incidence of human brucellosis is largely unknown in Latin American countries. In Argentina, for the period 1994-2006, B. melitensis was the principal cause of infection (145 cases), while B. suis caused substantial morbidity too (144 cases), almost twice as much as $B$. abortus (75 cases) [52]. B. suis was isolated in the vast majority of the cases in pigs but also in a significant number of cattle and dogs and the potential role of these animals as the source of $B$. suis for humans needs to be studied. Information on naturally occurring brucellosis in llama is very scarce, although experimentally llamas have been shown to be susceptible to B. abortus [53].

\section{Conclusions}

As far as the definition of a brucellosis "One Health" framework is concerned, it is of paramount importance to identify Brucella species infecting humans and the different animal species in order to rightly identify the source of infection and develop targeted control measures.

The following principles should always prevail:

- Although B. abortus may infect sheep and goats and B. melitensis cattle, only the preferential host species, i.e., sheep and goats for B. melitensis and cattle for B. abortus, act as reservoirs of infection and maintenance hosts. The situation in pigs is different, as pigs can act as maintenance hosts for B. abortus. Therefore, the Brucella species infecting animals has to be identified in order to advocate sound control measures in the maintenance host.

- The vaccination status always needs to be taken into consideration, particularly when studies rely on sero-prevalences, given that vaccines interfere with serology.

- Calves, lambs, kids and piglets, born from infected animals regardless of their vaccination status, may be infected even if they appear to be healthy. Those animals maintain the infection in the animal population even when vaccination is implemented.

- When mass vaccination is applied, both non-infected and infected animals are vaccinated. The protection conferred by preventive vaccination is well documented in reducing the abortion rate and the excretion of Brucella in milk, while the value of curative vaccination (i.e., vaccination of infected animals) of livestock remains to be studied, particularly its ability to reduce the number and the period of excretion of Brucella spp. in milk.

- The contribution of non-conventional livestock species (yacks and camels) to human brucellosis needs to be addressed. It is known that camelids of the old world can be infected with both B. melitensis and B. abortus, but their role as maintenance hosts 
needs to be studied.

- Humans can be infected with B. melitensis, B. abortus or B. suis. Identifying the animal reservoir, particularly in mixed herds or multi-species husbandry systems should always be done.

- Most human brucellosis cases have mainly two different origins: foodborne (milk and milk products) or occupational (farmer, butcher, veterinarian, etc.). If human cases are predominantly found in certain professional categories, it suggests that sanitary measures related to milk and milk products are well implemented, whereas control should be enhanced in the reservoir animal species. Conversely, should the majority of cases be found in the general population, this suggest that neither sanitary measures, nor control measures are efficiently implemented.

A "One Health" framework applied to brucellosis and other endemic zoonoses should encourage actors from the medical, veterinary, wildlife and sociological disciplines to contribute to a holistic understanding of the disease. Gross simplification of the real situation will not help. Professional, scientific and well documented contributions should result in consensual and effective control strategies, which should be seen as an important added value from a societal perspective.

\section{Conflict of interest}

None.

\section{References}

[1] Zinsstag J, Schelling E, Roth F, Bonfoh B, de Savigny D, Tanner M. Human benefits of animal interventions for zoonosis control. Emerging Infectious Diseases 2007; 13:527-31.

[2] Coker R, Rushton J, Mounier-Jack S, Karimuribo E, Lutumba P, Kambarage D, et al. Towards a conceptual framework to support one-health research for policy on emerging zoonoses. The Lancet Infectious Diseases 2011;11:326-31.

[3] Pappas G, Papadimitriou P, Akritidis N, Christou L, Tsianos EV. The newglobal map of human brucellosis. The Lancet Infectious Diseases 2006;6:91-9.

[4] Godfroid J, Cloeckaert A, Liautard JP, KohlerS, Fretin D, Walravens K, et al. From the discovery of the Malta fever's agent to the discovery of a marine mammal reservoir, brucellosis has continuously been a re-emerging zoonosis. Veterinary Research 2005;36:313-26.

[5] Xavier MN, Paixao TA, Poester FP, Lage AP, Santos RL Pathological, immunohistochemical and bacteriological study of tissues and milk of cows and fetuses experimentally infected with Brucella abortus. Journal of Comparative Pathology 2009;140:149-57.

[6] Glynn MK, Lynn TV. Brucellosis. Journal of the American Veterinary Medical Association 2008;233:900-8.

[7] Alexander KA, Blackburn JK, Vandewalle ME, Pesapane R, Baipoledi EK, Elzer PH. Buffalo bush meat, and the zoonotic threat of brucellosis in Botswana. PLoS ONE 2012;7:e32842.

[8] Ruben B, Band JD, Wong P, ColvilleJ. Person-to-person transmission of Brucella melitensis. The Lancet 1991;337:14-5.

[9] Marcotty T, Matthys F, Godfroid J, Rigouts L, Ameni G, van Pittius NG, et al. Zoonotic tuberculosis and brucellosis in Africa: neglected zoonoses or minor public-health issues? The outcomes of a multi-disciplinary workshop. Annals of Tropical Medicine and Parasitology 2009;103:401-11.

[10] Roth F, Zinsstag J, Orkhon D, Chimed-Ochir G, Hutton G, Cosivi O, et al. Human health benefits from livestock vaccination for brucellosis: case study. Bulletin of the World Health Organization 2003;81:867-76.

[11] Zinsstag J, Roth F, Orkhon D, Chimed-Ochir G, Nansalmaa M, Kolar J, et al. A model of animal-human brucellosis transmission in Mongolia. Preventive Veterinary Medicine 2005;69:77-95.

[12] Ward D, Jackson R, Karomatullo H, KhakimovT, Kurbonov K, Amir-bekov M, et al. Brucellosis control in Tajikistan using Rev 1 vaccine: change in seroprevalence in small ruminants from 2004 to 2009. Veterinary Record 2012;170:100.

[13] Coker AO, Isokpehi RD, Thomas BN, Fagbenro-Beyioku AF, Omilabu SA. Zoonotic infections in Nigeria: overview from a medical perspective. Acta Tropica 2000;76:59-63.

[14] Godfroid J, Scholz HC, Barbier T, Nicolas C, Wattiau P, Fretin D, et al. Brucellosis at the animal/ecosystem/human interface at the beginning of the 21st century. Preventive Veterinary Medicine 2011;102:118-31.

[15] Godfroid J, Saegerman C, Wellemans V, Walravens K, Letesson JJ, Tibor A, et al. How to substantiate eradication of bovine brucellosis when aspecific serological reactions occur in the course of brucellosis testing. Veterinary Microbiology 2002;90:461-77.

[16] Weynants V, Godfroid J, Limbourg B, Saegerman C, Letesson JJ. Specific bovine brucellosis diagnosis based on in vitro antigen-specific gamma-interferon production. Journal of Clinical Microbiology 1995;33:706-12.

[17] Moriyon I, Grillo MJ, Monreal D, Gonzalez D, Marin C, Lopez-Goni I, et al. Rough vaccines in animal brucellosis: structural and genetic basis and present status. Veterinary Research 2004;35:1-38.

[18] Nielsen K. Diagnosis of brucellosis by serology. Veterinary Microbiology 2002;90:447-59.

[19] Kabagambe EK, Elzer PH, Geaghan JP, Opuda-Asibo J, Scholl DT, Miller JE. Risk factors for Bruce/Zaseropositivity in goat herds in eastern and western Uganda. Preventive Veterinary Medicine 2001;52:91-108.

[20] Magona J, Walubengo J, Galiwango T, Etoori A. Seroprevalence and potential risk of bovine brucellosis in zerograzing and pastoral dairy systems in Uganda. Tropical Animal Health and Production 2009;41:1765-71.

[21] Makita K, Fevre E, Waiswa C, Eisler M,Thrusfield M, Welburn S. Herd prevalence of bovine brucellosis and analysis of risk factors in cattle in urban and periurban areas of the Kampala economic zone, Uganda. BMC Veterinary Research 2011;7:60.

[22] Makita K, Fevre EM, Waiswa C, Kaboyo W, De Clare Bronsvoort BM, Eisler MC, et al. Human brucellosis in urban and peri-urban areas of Kampala, Uganda. Annals of the New York Academy of Sciences 2008;1149:309-11.

[23] Bernard F, Vincent C, Matthieu L, David R, James D. Tuberculosis and brucellosis prevalence survey on dairy cattle in Mbarara milk basin (Uganda). Preventive Veterinary Medicine 2005;67:267-81.

[24] Makita K, Fevre EM, Waiswa C, Eisler MC, Welburn SC. How human brucellosis incidence in urban Kampala can be reduced most efficiently? A stochastic risk assessment of informally-marketed milk. PLoS ONE 2010;5:e14188.

[25] Makita K, Fevre E, Waiswa C, Kaboyo W, Eisler M, Welburn S. Spatial epidemiology of hospital-diagnosed brucellosis in Kampala, Uganda. International Journal of Health Geographics 2011;10:52.

[26] Meyer ME, Morgan WJB. Designation of neotype strains and of biotype reference strains for species of the genus Brucella Meyer and Shaw. International Journal of Systematic Bacteriology 1973;23:135-41.

[27] Muendo E, Mbatha P, Macharia J, Abdoel T, Janszen P, Pastoor R, et al. Infection of cattle in Kenya with Brucella abortus biovar3 and Brucella me//tens/s biovar1 genotypes. Tropical Animal Health and Production 2012;44:17-20.

[28] Mwebe R, Nakavuma J, Moriyon I. Brucellosis seroprevalence in livestock in Uganda from 1998 to 2008: a retrospective study. Tropical Animal Health and Production 2011;43:603-8.

[29] Verger JM, Garin-Bastuji B, Grayon M, Mahe AM. Brucella-melitensis infection in cattle in France. Annales de Recherches Veterinaires 1989;20:93-102. 
[30] Alvarez J, Suaez JL, Garcia N, Serrat C, Perez-Sancho M, Gonzalez S, et al. Management of an outbreak of brucellosis due to B. meliten-sis in dairy cattle in Spain. Research in Veterinary Science 2011;90: 208-11.

[31] Riber U, Jungersen G. Cell-mediated immune responses differentiate infections with Brucella suis from Yersinia enterocolitica serotype 0:9 in pigs. Veterinary Immunology and Immunopathology 2007;116:13-25.

[32] McDermott JJ, ArimiSM. Brucellosis in sub-Saharan Africa: epidemiology, control and impact. Veterinary Microbiology 2002;90:111-34

[33] Diaz R, Casanova A,Ariza J, Moriyon I. The Rose Bengal Test inhuman brucellosis: a neglected test for the diagnosis of a neglected disease. PLoS Neglected Tropical Diseases 2011;5:e950.

[34] Zhang WY, Guo WD, Sun SH, Jiang JF, Sun HL, Li SL, et al. Human brucellosis, Inner Mongolia, China. Emerging Infectious Diseases 2010;16:2001-3.

[35] Minas A, Minas M, Stournara A, Tselepidis S. The effects of Rev-1 vaccination of sheep and goats on human brucellosis in Greece. Preventive Veterinary Medicine 2004;64:41-7.

[36] Ocholi RA, Kwaga JKP, Ajogi I, Bale JOO. Phenotypic characterization of Brucella strains isolated from livestock in Nigeria. Veterinary Microbiology 2004; 103:47-53.

[37] Holt HR, Eltholth MM, Hegazy YM, El-Tras WF, Tayel AA, Guitian J. Brucella spp. infection in large ruminants in an endemic area of Egypt: cross-sectional study investigating seroprevalence, risk factors and livestock owner's knowledge, attitudes and practices (KAPs). BMC Public Health 2011:11.

[38] Bekele M, Mohammed H, Tefera M, Tolosa T. Small ruminant brucellosis and community perception in Jijiga District, Somali Regional State Eastern Ethiopia. Tropical Animal Health and Production 2011;43:893-8.

[39] Stoffregen WC, Olsen SC, Wheeler CJ, Bricker BJ, Palmer MV, Jensen AE, et al. Diagnostic characterization of a feral swine herd enzootically infected with Brucella. Journal of Veterinary Diagnostic Investigation 2007;19:227-37.

[40] Godfroid J. Brucellosis in wildlife. Revue Scientifiqueet Technique de 1 Office International des Epizooties 2002;21:277-86

[41] Van Campen H, Rhyan J. The role of wildlife in diseases of cattle. Veterinary Clinics of North America-Food Animal Practice 2010;26, 147+.

[42] Waghela S, Karstad L. Antibodies to Brucella spp. among blue wildebeest and African buffalo in Kenya. Journal of Wildlife Diseases 1986;22:189-92.

[43] Meyer ME. Species identity and epidemiology of Brucella strains isolated from Alaskan Eskimos. Journal of Infectious Diseases 1964;114:169.

[44] Forbes LB. Isolates of Brucella-Suis Biovar-4 from animals and humans in Canada, 1982-1990. Canadian Veterinary Journal-Revue Veterinaire Canadienne 1991;32:686-8.

[45] Gwida M, El-Gohary A, Melzer F, Khan I, Rösler U, Neubauer H. Brucellosis in camels. Research in Veterinary Science 2011.

[46] Musa MT, Eisa MZM, El Sanousi EM, Wahab MBA, Perrett L. Brucellosis in camels (Camelus dromedarius) in Darfur, Western Sudan. Journal of Comparative Pathology 2008; 138:151-5.

[47] Jones KE, Patel NG, Levy MA, Storeygard A, Balk D, Gittleman JL, et al. Global trends in emerging infectious diseases. Nature 2008;451:990-3.

[48] Samaha H, Al-Rowaily M, Khoudair RM, Ashour HM. Multicenter study of brucellosis in Egypt. Emerging Infectious Diseases 2008;14:1916-8.

[49] Matope G, Bhebhe E, Muma J, Skjerve E, Djonne B. Characterization of some Brucella species from Zimbabwe by biochemical profiling and AMOS-PCR. BMC Research Notes 2009;2:261.

[50] Franco MP, Mulder M, Gilman RH, Smits HL Human brucellosis. The Lancet Infectious Diseases 2007:7:775-86.

[51] Al Dahouk S, Nockler K. Implications of laboratory diagnosis on brucellosis therapy. Expert Review of Anti-Infective Therapy 2011;9:833-45.

[52] Lucero NE, Ayala SM, Escobar GI, Jacob NR. Brucella isolated in humans and animals in Latin America from 1968 to 2006 . Epidemiology and Infection 2008; 136:496-503.

[53] Gilsdorf MJ, Thoen CO, Temple RMS, Gidlewski T, Ewalt D, Martin B, et al. Experimental exposure of llamas (Lama glama) to Brucella abortus: humoral antibody response. Veterinary Microbiology 2001;81:85-91. 